INPLASY

PROTOCOL

To cite: Zou et al. Fire needle therapy for blood stasis syndrome of plaque psoriasis :

A protocol for systematic review and meta-analysis. Inplasy protocol 202120007. doi:

10.37766/inplasy2021.2.0007

Received: 01 February 2021

Published: 02 February 2021

Corresponding author: Jiahua Zou

570136847@qq.com

Author Affiliation: Jiangxi University of Traditional Chinese Medicine

Support: NO:2018YFC1705303.

Review Stage at time of this submission: Preliminary searches.

Conflicts of interest: None declared.

\section{Fire needle therapy for blood stasis syndrome of plaque psoriasis : A protocol for systematic review and meta-analysis}

Zou,JH; Huang, G; Hu, CX; Huang, DB4; Huang, Q5; Chen, PZ6; Yan, J7; Zhang, FY8; Gong, LP9.

Review question / Objective: This study will provide high quality evidence based medicine to evaluate the effectiveness and safety of fire needle for plaque psoriasis (blood stasis syndrome), and further seek its scientific and effective Chinese medicine treatment methods.

Condition being studied: Plaque psoriasis(PSO) is a common clinical chronic inflammatory skin disease. Its typical features are mainly scaly erythema or plaque, accompanied by different with a degree of itching. Moreover, a rash can occur anywhere on the body, such as the scalp, elbows, knees, waist and trunk. Patients with psoriasis are not only greatly affected by their appearance and social life, but also bear heavy psychological pressure.The incidence rate is increasing year by year due to the fast pace of work and unhealthy diet. Fire needle has been widely used in the treatment of PSO. However, the efficacy of fire needle for PSO is uncertain. Thus, the purpose of this systematic review is to evaluate the effectiveness and safety of fire needle for PSO (blood stasis syndrome).

INPLASY registration number: This protocol was registered with the International Platform of Registered Systematic Review and Meta-Analysis Protocols (INPLASY) on 02 February 2021 and was last updated on 02 February 2021 (registration number INPLASY202120007).

\section{INTRODUCTION}

Review question / Objective: This study will provide high quality evidence based medicine to evaluate the effectiveness and safety of fire needle for plaque psoriasis (blood stasis syndrome), and further seek its scientific and effective Chinese medicine treatment methods.

Condition being studied: Plaque psoriasis(PSO) is a common clinical chronic inflammatory skin disease. Its typical features are mainly scaly erythema 
or plaque, accompanied by different with a degree of itching. Moreover, a rash can occur anywhere on the body, such as the scalp, elbows, knees, waist and trunk. Patients with psoriasis are not only greatly affected by their appearance and social life, but also bear heavy psychological pressure.The incidence rate is increasing year by year due to the fast pace of work and unhealthy diet. Fire needle has been widely used in the treatment of PSO. However, the efficacy of fire needle for PSO is uncertain. Thus, the purpose of this systematic review is to evaluate the effectiveness and safety of fire needle for PSO (blood stasis syndrome).

\section{METHODS}

Search strategy: Searching through a combination of medical subject headings and text words.Key words include psoriasis, psoriasis vulgaris, plaque psoriasis, BaiBi, fire needle therapy, fire needle, burning red acupuncture, fire acupuncture, randomized controlled trials, controlled clinical trials, clinical trials, trials. Table 1 specifically shows Pubmed's search strategy. At the same time, the search method is appropriately modified according to the difference between the Chinese database and the English database.

Participant or population: The Participants in the study must meet both the clinical diagnostic criteria for PSO and the blood stasis syndrome of TCM.There are no restrictions on race, age, gender, lifestyle, education level.

Intervention: Single fire needle interventions and fire needle as the main part of the combined therapy Such as fire needle combined with traditional chinese herbal, western medicine,308-nm excimer laser, cupping will be included. There will be no limited to the fire needle materials or frequency of intervention.

Comparator: Different types of interventions will be included (e.g single western medicine,traditional chinese herbal,308-nm excimer laser,other combination therapy, etc).Other types of interventions such as fire needle will be excluded.

Study designs to be included: All RCTs of fire needle treatment for PSO(blood stasis syndrome) will be included, regardless of whether blind method is used.Other types of studies such as non-RCTs will be excluded.

Eligibility criteria: All RCTs of fire needle treatment for PSO(blood stasis syndrome) will be included, regardless of whether blind method is used. Other types of studies such as non-RCTs will be excluded. Exclusion criteria as follows: (1)Select the latest one among the repeated publications; (2)Women in a special period (pregnancy or lactation); (3)Non blood stasis syndrome; (4)Joint psoriasis, Pustular psoriasis, Erythroderma psoriasis; (5)Documents whose full text cannot be obtained from various sources; (6)Combined with other serious organic diseases or mental diseases.

Information sources: The following electronic databases will be searched from inception to October 2020: PubMed, Web of Science, Embase, Cochrane Library, China National Knowledge Infrastructure, WangFang Database, Chinese Science Journal Database, Chinese Biomedical Literature Database. In addition, other documents that meet the requirements will be manually searched, including conference papers, dissertations, etc.

Main outcome(s): The Primary outcomes include clinical efficacy, psoriasis area and severity index (PASI). 1.The total clinical effective rate is obtained by adding the cure rate and effective rate;2.PASI score, as an important indicator for evaluating the severity of psoriasis skin manifestations, is stable and reliable.

Additional outcome(s): 1.Itchy(VAS); 2.Symptom score according to the evaluation standard of Chinese medicine; 3.Dermatological quality of life index (DLQI); 4.Adverse events, such as infection, 
redness, burning, blisters, pigmentation, itching, etc.

Data management: The data will be extracted independently by the 2 researchers, then cross-check and verify each other.Any disagreement will be resolved by discussion with a third reviewer.The extracted data mainly includes the following information:literature information (first author, publication year, study area),research methods, participant characteristics, sample size, intervention(s), outcome(s), adverse event(s), and other relevant characteristics.

Quality assessment / Risk of bias analysis: Two researchers will use the Cochrane risk assessment tool to evaluate the included literature, including the following 7 aspects:random sequence generation, allocation concealment, blinding of participants and researchers and outcome assessors, incomplete outcome data, selective outcome reporting, and other bias. The assessments will be classified into 3 levels:low risk, high risk, and unclear risk.Selecting "yes" indicates that there is a low risk;"no" indicates that there is a high risk; "unclear" indicates that there is a risk of uncertain deviation. Issues are resolved by rechecking the Original document and further discussions with the third reviewer.

Strategy of data synthesis: RevMan software(Version5.3, Copenhagen:The Nordic Cochrane Centre,The Cochrane Collaboration,2014)will be applied in this meta-analysis. When the analysis data is continuous data, results will be reported as mean difference(MD) or standard mean difference(SMD) with $95 \%$ confidence interval $(95 \% \mathrm{Cl})$.the dichotomous data will be calculated with the odds ratio (OR) with 95\% Cl.We will detect the heterogeneity between studies by Chi-Squared test and Higginsl2 test.When $P>0.1$ andl2 $<50 \%$, there is no obvious heterogeneity and the fixed-effects model will be used; Otherwise, the random effects model will be used for merger the analysis. When there is significant clinical heterogeneity between studies, descriptive analysis will be performed.
Subgroup analysis: If significant heterogeneity is detected in all studies, we will try to conduct a subgroup analysis based on patient characteristics, interventions (including simple fire needles, fire needles combined with different drugs or physical therapies) and treatment time.

Sensitivity analysis: If necessary, sensitivity analysis is performed according to the sample size and methodology of the research to obtain a stable and reliable result.

Language: English.

Country(ies) involved: China.

Keywords: fire needle; plaque psoriasis; blood stasis syndrome; protocol; systematic review.

Contributions of each author:

Author 1 - Jiahua Zou Conceptualization; Data curation; Investigation; Methodology;Project administration; Writing-original draft.

Author 2 - Gang Huang Conceptualization; Investigation.

Author 3 - ChuXiang Hu - Formal analysis; Software.

Author 4 - Dongbei Huang - Formal analysis.

Author 5 - Qiang Huang - Formal analysis.

Author 6 - Peize Chen - Formal analysis.

Author 7 - Juan Yan - Data curation; Project administration; software.

Author 8 - FeiYan Zhang - Data curation; Writing-original draft.

Author 9 - Liping Gong - Conceptualization; Supervision;Writing-review\&editing. 